\title{
Some features of transverse photovoltage in semiconductor heterostructure and Schottky contact
}

\author{
L.V. Shekhovtsov \\ Institute of Semiconductor Physics, National Academy of Sciences of Ukraine, \\ Prospect Nauky 45, 03028 Kyiv, Ukraine \\ Phone: 38044 2659464,Fax: 38044 2658342,E-mail:k208@photon.kiev.ua
}

\begin{abstract}
We investigated transverse photovoltage generated in semiconductor heterostructures by a modulated illumination. A complicated form of the photovoltage spectral curves for Ge-GaAs heterostructures and $\mathrm{NbN}-\mathrm{GaAs}$ Schottky contacts is determined by interaction between photocurrents flowing in the bulk and near interfaces.

An additional unmodulated illumination of the samples during the measurements of the photovoltage spectral curves changes the interaction of photocurrents. This leads to modification of the photovoltage spectral curves. An analysis of their features enables one to put forward models for interaction of photocurrents flowing near the interfaces and obtain the characteristics of the interface uniformity in composite semiconductor structures.
\end{abstract}

Keywords: heterostructure, Schottky contact, transversal photovoltage, inhomogeneity.

Paper received 07.08.01; revised manuscript received 16.10.01; accepted for publication 12.12.01.

\section{Introduction}

When a metal-semiconductor (or semiconductor-semiconductor) physical contact is formed, then, in almost all cases, a nonuniform band bending [ $1-5]$ or nonuniform transition layer at the interface $[6,7]$ appears. These processes were observed for various contacts ( $\mathrm{Au}-\mathrm{Si}, \mathrm{Au}-\mathrm{GaSe}, \mathrm{Al}-\mathrm{GaS}$, $\mathrm{Au}-\mathrm{SiN}_{\mathrm{X}}-\mathrm{GaAs}, \mathrm{Si}^{-\mathrm{SiO}_{2}}$ ) and were studied using different techniques, such as ballistic electron emission [1,2], temperature dependence of current-voltage characteristics [3], photoemission-spectromicroscopy [4,5], scanning near-field optical microscopy [6], medium energy ion scattering spectroscopy [7], etc.

The obtained experimental results give impetus to discussion about mechanisms for formation of the above nonuniformities both at clean semiconductor surfaces [8] and in metal-semiconductor contacts [9].

It is known that illumination of a semiconductor crystal with non-uniformly distributed impurities generates a bulk photovoltage in it. This photovoltage has a component oriented along the interface [10]. A non-uniformly doped semiconductor layer, as well as thickness fluctuations of a metal film, in their turn, may cause a band bending nonuniformity in metal-semiconductor contacts $[9,11,12]$.

Here a unique photoelectric technique is used, namely, we study spectral characteristics of the transverse photovoltage in Schottky contact and the semiconductor heterostructure that stems from the above nonuniformities. A technique to measure an alternating photovoltage that occurs along the interfaces of semiconductor structures (e.g., Ge-GaAs heterostructure or NbN-GaAs Schottky contact) involves concurrent illumination of a sample with modulated and unmodulated monochromatic light beams. By changing the unmodulated light wavelength 1(i.e., by changing coefficients of its absorption in $\mathrm{Ge}$ and $\mathrm{GaAs}$ ), one can change the nonequilibrium charge carrier concentration in the heterostructure layers and, correspondingly, conductivity of these layers.

By probing the sample studied with modulated light beam and simultaneously changing its wavelength, one can obtain spectral characteristics for photovoltage. Their features depend also on the unmodulated light wavelength, that serves as a parameter.

An analysis of spectral characteristics for the bulk-gradient transverse photovoltage [10] generated in Ge-GaAs- 


\section{L.V. Shekhovtsov: Some features of transverse photovoltage...}

type heterostructures or NbN-GaAs Schottky contacts under illumination with monochromatic light on the film $(\mathrm{Ge}, \mathrm{NbN})$ or substrate (GaAs) sides allows to find if transition layer that may appear during contact formation is present.

Besides, it enables one to refine such characteristics as resistivity distribution over the semiconductor structure studied.

Both Ge-GaAs-type heteroepitaxial structures and Schottky contacts can serve as a good model to demonstrate efficiency of the above technique.

It is known that, as a rule, semiconductor crystals have native resistivity nonuniformities. Additional nonuniformities may appear near interfaces when contacts of various types are formed. It should be noted that we took no special steps aimed at formation of nonuniformities or gradients of doping in the samples studied.

\section{Ge-GaAs heterostructure}

\subsection{Experimental details}

Shown in Fig. 1 is the diagram of the Ge-GaAs heterostructure sample used in our experiment. The thickness of the $p$-Ge film $\left(p=10^{16} \mathrm{~cm}^{-3}\right)$ was $0.1-0.2 \mu \mathrm{m}$; that of semiinsulating $\mathrm{GaAs}$ substrate was $300 \mu \mathrm{m}$. The contacts $C_{1}$ and $C_{2}$ were deposited on the film side; the intercontact spacing was $12-15 \mathrm{~mm}$. The sample width was from 2 to $3 \mathrm{~mm}$; the illuminated part of the sample $(l \times w)$ was $(5 \times$

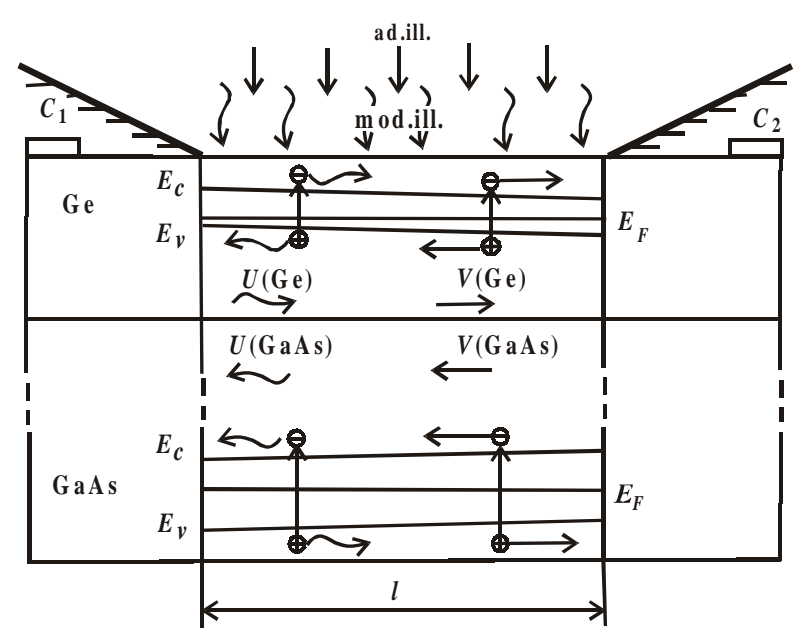

Fig. 1. Geometry of experiment and mechanism for transverse photovoltage appearance in the Ge-GaAs heterostructure:

$U(\mathrm{Ge})$ and $U(\mathrm{GaAs})$ - the transverse photovoltage generated in the Ge film and GaAs substrate, respectively, under modulated illumination; $V$ - the transverse voltage that appears in the Ge film under additional unmodulated illumination; $l$ - the length of the illuminated sample section; $E_{C}$ - the conduction band bottom; $E_{V}-$ the valence band top; $E_{F}-$ the Fermi level; $C_{1}$ and $C_{2}-$ the measuring contacts; mod. ill. - modulated monochromatic illumination; add. ill. - unmodulated monochromatic illumination. $\times 2) \mathrm{mm}$, the width $w$ of the illuminated part being not indicated in Figs 1 and 3.

Some steps have been taken to exclude contact illumination with exciting radiation during measurements. To prevent generation of stray barrier photovoltage, the sample near-contact regions have been screened with a black nontransparent material. The spacing between the boundaries of the illuminated part of the sample and contacts was $3 \mathrm{~mm}$. This is far in excess of diffusion length for nonequilibrium electron-hole pairs in both Ge (hundreds of microns) and GaAs (tens of microns). Illumination was realized on the film side normally to the sample surface. The modulated monochromatic light beam and additional unmodulated light beam illuminated the same section of the sample.

Images of slots from two monochromators were focused on the sample surfaces. These images served as sources of modulated and unmodulated radiation. They were light beams of rectangular cross section ( $22 \mathrm{~mm}$ long by $2 \mathrm{~mm}$ wide). The above length ( $22 \mathrm{~mm}$ ) was over that of the illuminated part of the sample. The light intensity was the same over the whole cross section.

The alternating photovoltage $U$ was measured across the $C_{1}$ and $C_{2}$ contacts at the light modulation frequency of $538 \mathrm{~Hz}$. Use of synchronous detection enabled us to note when the photovoltage relative polarity reversed. Our technique made it possible also to study photovoltage distribution along the sample using a movable monochromatic light probe $0.3 \mathrm{~mm}$ wide. We could change both the velocity of probe movement and wavelength of exciting radiation, i.e., the depth of light absorption in the semiconductor structure.

The measurements were performed at room temperature.

In Fig. 1 the resistivity gradients in the Ge film and GaAs substrate (due to nonuniform doping of the heterostructure regions adjacent to the interface) are shown, by convention, as slopes of the Ge conduction band bottom, $\mathrm{E}_{\mathrm{C}}$, and $\mathrm{GaAs}$ valence band top, $\mathrm{E}_{\mathrm{V}}$, levels, respectively. It should be noted that presence of a smooth resistivity gradient of the same sign over the whole illuminated area is not necessary for bulk-gradient photovoltage to appear. It would be sufficient that such a gradient (or its component) was present in the crystal region whose size is comparable to (or bigger than) the diffusion length of the nonequilibrium electron-hole pairs.

In Fig. 1 such pairs (generated by modulated illumination) are shown, by convention, as circles with wavy arrows, while the nonequilibrium charge carriers (generated by the additional unmodulated illumination) are shown as circles with straight arrows.

Relative polarities of voltages $\mathrm{U}$ and $\mathrm{V}$ are determined by the direction of charge carriers motion, i.e., the orientation of the same resistivity gradient (band slope) in Ge and GaAs, respectively. It is evident that both polarities are the same. This is shown in Fig. 1 for $\mathrm{U}(\mathrm{Ge})$ and $\mathrm{V}(\mathrm{Ge})$ (as well as for $\mathrm{U}(\mathrm{GaAs})$ and $\mathrm{V}(\mathrm{GaAs})$ ) by arrows oriented in the same direction. 


\section{L.V. Shekhovtsov: Some features of transverse photovoltage...}

\subsection{Discussion of results}

Shown in Fig. 2 are the representative transverse photovoltage U spectral curves taken for the Ge-GaAs structure. Curve 1 corresponds to the case without additional unmodulated illumination. The intensity $G$ of modulated illumination was the same $\left(2 \times 10^{14}\right.$ photons $\left./ \mathrm{cm}^{2} \mathrm{~s}\right)$ over the whole spectral range used.

The physical reasons for the complicated photovoltage spectral curve are related to the fact that the resistivity gradients of the film and substrate along the interface are oppositely directed [13].

This is evidenced by the experimental studies of photovoltage distribution made with moving monochromatic light probe. The type of distribution was different for photovoltage of a definite polarity, and polarity reversed when the wavelength changed from $\lambda=0.65 \mu \mathrm{m}$ to $0.89 \mu \mathrm{m}$.

This is related to the fact that a transition layer of variable thickness may be formed between Ge and GaAs. In this case a nonuniform band bending and variable SCR thickness are realized along the interface. As a result, the near-interface layers of semiconductors that form a contact have different conductivities.

In the wavelength range $\lambda=(0.54-0.7) \mu \mathrm{m}$, the photovoltage $U(\mathrm{Ge})$ is generated predominantly in the $\mathrm{Ge}$ film. As the wavelength $\lambda$ grows, and the coefficient of light absorption in germanium decreases, the modulated light becomes absorbed predominantly in the GaAs substrate (where the resistivity gradient is oriented oppositely to that in Ge film). As a result, the photovoltage $U(\mathrm{GaAs})$ reverses its sign (Fig. 2, curve 1). Curve 2 was taken under additional unmodulated illumination of wavelength $\lambda=0.68 \mu \mathrm{m}$ (the corresponding photon energy $h v=1.82 \mathrm{eV}$ ) and intensity $G=2 \times 10^{14}$ photons $/ \mathrm{cm}^{2}$ s, i.e., the same as that of modulated illumination.

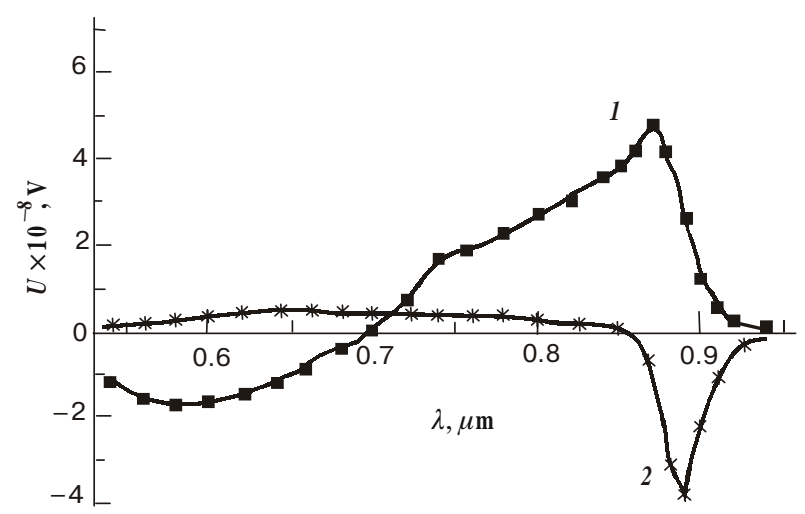

Fig. 2. Spectral characteristics of the transverse photovoltage measured for the Ge-GaAs heterostructure at the modulated illumination intensity $G_{\mathrm{MOD}}=2 \times 10^{14}$ photon $/ \mathrm{cm}^{2} \mathrm{~s}$ :

1 - without additional unmodulated illumination;

2 - at additional unmodulated illumination of intensity $\mathrm{G}_{\mathrm{ADD}}=$ $=2 \times 10^{14}$ photon $/ \mathrm{cm}^{2} \mathrm{~s}$.
For additional monochromatic illumination with wavelength $\lambda=0.68 \mu \mathrm{m}$ the coefficient of light absorption $\alpha$ in Ge is $10^{5} \mathrm{~cm}^{-1}$ [14]. When Ge film thickness $d$ is $(0.1-0.2) \mu \mathrm{m}$, then this light is absorbed predominantly in the film. Here a continuous photovoltage $V$ is generated. The corresponding electric field is oriented along the interface. It affects the movement of the electron-hole pairs generated by the modulated light beam in the GaAs substrate close to the interface.

The photovoltage spectral characteristic taken under the above additional illumination shows a change: the photovoltage peak near the GaAs intrinsic absorption edge reverses its sign (Fig. 2, curve 2). There is some kind of a photovoltage «surge» of the opposite sign. It is obvious that the additional illumination wavelength, at which the above effect is observed, depends on the Ge film thickness (the sample was illuminated on the side of this film).

Similar photovoltage «surge» was also observed when the sample was illuminated on the GaAs side.

The reason for the above effect is that photoconducti- vity (at a light modulation frequency) arises in the field $E$ generated by the unmodulated additional illumination [15].

This additional illumination leads to appearance of a continuous (unmodulated) bulk-gradient photovoltage and, as a result, additional continuous field in the sample. In this case, when the sample is illuminated with a modulated light beam in the semiconductor intrinsic absorption region, then a voltage appears in the above field: $U_{\mathrm{ADD}}=-V \times R \times \Delta \sigma$.

Here $V=E \times l$ is the photovoltage across $C_{1}$ and $C_{2}$ contacts generated by the continuous additional illumination; $l$ is the length of the illuminated part of the sample; $R$ is resistance of this part, $\Delta \sigma$ is photoconductance appearing due to the modulated illumination. $E$ is the direct electric field generated by the additional illumination.

It is apparent that, depending on the values of coefficients of modulated and unmodulated light absorption in a heterostructure, a situation may occur when the additional voltage due to photoconductivity, as well as the transverse photovoltage, are generated (at a modulation frequency) in both the GaAs substrate and Ge film. The resulting voltage, being an algebraic sum of the two above components, may change its polarity, depending on the ratio between their amplitudes. This is just the effect we have observed experimentally.

When explaining the obtained spectral characteristics of transverse photovoltage, one should analyze possible contribution to the measured signal from the «lateral» photovoltage. It may be generated along the interface between semiconductor layers of different conductivity values [16].

Such voltage can be photogenerated when the illuminated part of semiconductor structure is located asymmetrically relatively to the measuring contacts, as well as when a nonuniform band bending exists either near the surface or at the interface of the semiconductor structure studied. A nonuniform distribution of the light probe intensity can also contribute to such voltage. The necessary condition for the «planar» voltage generation is nonequilibrium charge carrier injection from one layer to another. 


\section{L.V. Shekhovtsov: Some features of transverse photovoltage...}

It is known that near semiconductor surface both band bending and, correspondingly, SCR (not shown in Fig. 1) exist. For Ge-GaAs heterostructure there is band bending at the interfaces too. Thus, in the samples studied, there are formally the conditions for generation of such voltage.

Our previous investigations have shown, however, that the main contribution comes from the voltage related to resistivity gradients. They appear during the Ge film growth on GaAs substrate [13].

First, of all, the voltage did not reverse its polarity when the light probe moved along the sample (such change should occur at generation of «lateral» voltage). In addition, the dependence of voltage amplitude on the exciting illumination intensity was nonmonotonous.

First the amplitude grew with light intensity, but then it abruptly dropped. Similar changes of amplitude were observed when the intensity of unmodulated additional illumination increased. These features are related to the presence of recombination-drift barriers in the bulk and nearsurface layers. Such barriers appear in heavily doped or compensated semiconductors. As was stated above, similar voltage «surge» is also observed when the structure is illuminated with unmodulated light on the GaAs substrate side.

Thus, one can conclude that the principal effect on the features of spectral characteristics of transverse voltage stems from the gradients of dark charge carriers. In this case, possible contribution to the measured signal from the «planar» voltage is less than that from the bulk voltage.

Let us discuss the mechanism for the effect of continuous field (that is generated by the additional illumination in both the Ge film and GaAs substrate) on the alternating photocurrent.

When a structure is illuminated with unmodulated light, then a direct voltage across $C_{1}$ and $C_{2}$ always appear. Both amplitude and polarity of this potential difference are determined by the voltage generation mechanism.

In general, the voltage is an algebraic sum of amplitudes of voltages generated in different layers of semiconductor structure. It is of importance that generation of photovoltage by modulated radiation occurs when the above direct voltage is present in the sample.

Thus, an unmodulated additional illumination may affect the conditions for current flow in the structure layers in two ways.

On the one hand, an increase of charge carrier concentration in a semiconductor at unmodulated additional illumination results in band bending changing at semiconductor surface or structure interface.

On the other hand, a direct voltage generated, say, in the semiconductor structure bulk is applied to the whole structure and affects conditions of current flow near the interface or in SCR.

A distinguishing feature is that amplitude of the photovoltage «surge» drops abruptly when the additional illumination wavelength is increased or decreased relative to the value $\lambda=0.68 \mu \mathrm{m}$. Wavelength decrease results in absorption of the additional illumination in the Ge film, while a wavelength increase leads to a growth of the absorbed light in the GaAs substrate farther from the interface. Therefore, if the additional continuous field is generated near the interface, then it substantially affects the alternating voltage. From this, one may conclude that the peculiarities of the transverse photovoltage spectral curve are determined by the properties of those film and substrate layers that are adjacent to the interface. The above peculiarities are formed due to absorption of modulated light in these layers.

\section{NbN-GaAs Schottky contact}

\subsection{Experimental details}

For a metal-semiconductor NbN-GaAs structure the spectral curve of the transverse photovoltage has some specific features.

The typical curves presented in Fig. 3 were taken for the structure where the $\mathrm{NbN}$ film thickness was $0.01 \mathrm{~mm}$ and that of the n-GaAs substrate was $200 \mathrm{~mm}$. It should be noted that there is a big $(0.5-0.8) \mathrm{eV}$ [17] depletion band bending near the interface of the $\mathrm{NbN}-\mathrm{GaAs}$ Schottky contact. At the dark charge carrier concentration of $5 \times 10^{16} \mathrm{~cm}^{-3}$ in $n$-GaAs the space charge region (SCR) width, $L_{\mathrm{SCR}}$, is about $0.2 \mu \mathrm{m}$.

The samples were $(10 \times 12) \mathrm{mm}$ rectangles. The illuminated part of the sample $(l \times w)$ was $(5 \times 2) \mathrm{mm}$. Illumination of the sample with both modulated and unmodulated light beams was performed on the GaAs substrate side. For both beams the illumination intensity was $8 \times 10^{14}$ photons $/ \mathrm{cm}^{2} \mathrm{~s}$. As in the case of Ge-GaAs heterostructure, the modulated illumination intensity was the same over the whole spectral range studied.

The near-contact areas were covered with a nontransparent material, just as it has been done when measuring voltage for Ge-GaAs heterostructure samples.

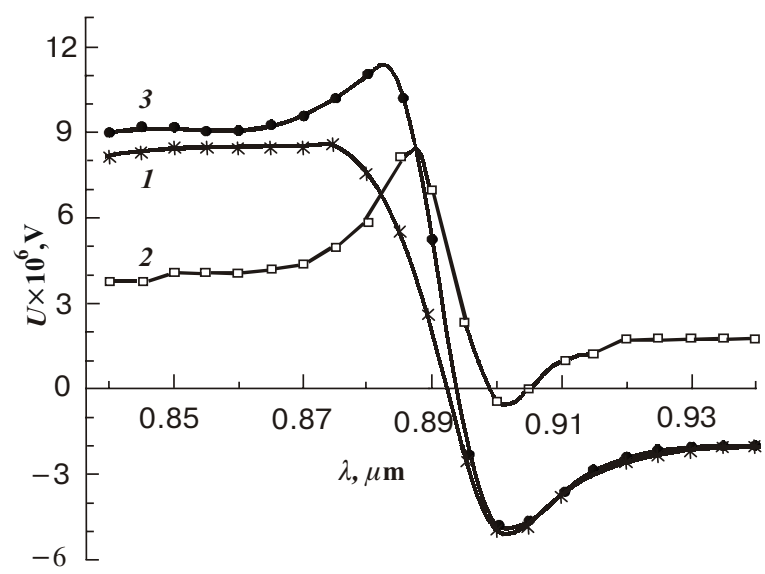

Fig. 3. Spectral characteristics of the transverse photovoltage measured for the $\mathrm{NbN}-\mathrm{GaAs}$ Schottky contact at the modulated illumination intensity $G_{\mathrm{MOD}}=8 \times 10^{14}$ photon $/ \mathrm{cm}^{2} \mathrm{~s}$ :

1 - without additional unmodulated illumination;

2,3 - at additional unmodulated illumination of intensity $G_{\mathrm{ADD}}=$ $=8 \times 10^{14}$ photon $/ \mathrm{cm}^{2} \mathrm{~s}$. 


\section{L.V. Shekhovtsov: Some features of transverse photovoltage...}

One can see from Fig. 3 that without illumination the photovoltage changes its sign near the GaAs intrinsic absorption edge (Fig. 3, curve 1) and then goes down smoothly at photon energies below the GaAs gap. Curve 2 was taken under the monochromatic $(\lambda=0.88 \mu \mathrm{m}$, photon energy $h v=1.41 \mathrm{eV})$ illumination. In this case the photovoltage was of the same polarity over the whole light wavelength range. Its amplitude dropped in the $\lambda=$ (0.64-0.86) $\mu \mathrm{m}$ range.

Curve 3 corresponds to the photovoltage spectral characteristic taken under additional illumination with $\lambda=$ $0.92 \mu \mathrm{m}$ (photon energy $h v=1.35 \mathrm{eV}$ ). The photovoltage value at $\lambda=0.85 \mu \mathrm{m}(h v=1.46 \mathrm{eV})$ increased under this illumination as compared to the curve 1 .

We have also measured spectral characteristics of the transverse voltage for the Schottky contact samples illuminated on the $\mathrm{NbN}$ film side. The features of these characteristics, however, were pronounced but slightly, and the amplitude of measured signal was very small. This seems to result from the fact that most of exciting radiation was absorbed in the film.

\subsection{Discussion of results}

The spectral curves measured for the $\mathrm{NbN}-\mathrm{GaAs}$ structure may be explained assuming that there exist nonuniformities of both GaAs bulk doping and band bending along the NbN-GaAs interface (see Fig. 4).

A sort of correlation is to exist between the nonuniformities of the bulk doping level and depletion band bending [9]. When the bulk concentration of charge carriers in the dark is decreased (increased), then the above band bending grows (drops).

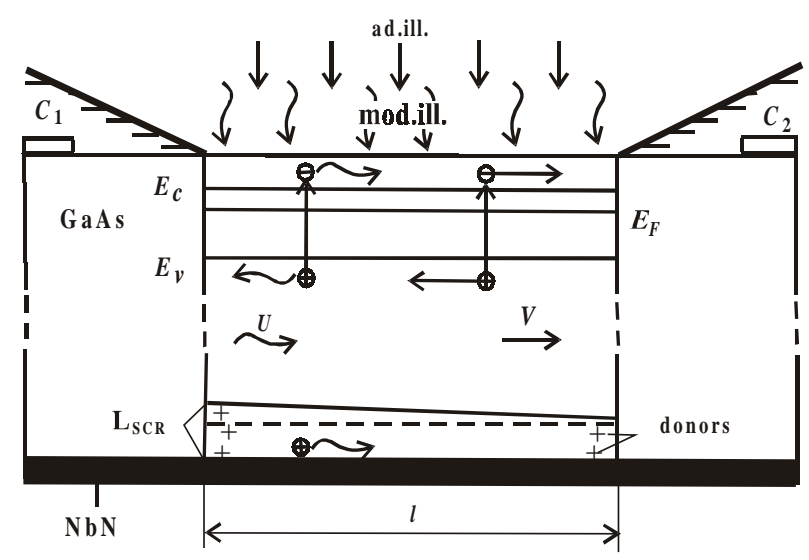

Fig. 4. Mechanism for transverse photovoltage generation in the NbN-GaAs Schottky contact:

$U$ - the transverse photovoltage generated in the GaAs substrate under modulated illumination; $V$ - the transverse voltage that appears in the GaAs substrate under additional unmodulated illumination; $L_{\mathrm{SCR}}$ - the space charge region width; (for other notions see Fig.1).
It was noted earlier that band bending nonuniformity might be affected also by the metal film thickness variations along the interface $[11,12]$. This means that the gradient of positively charged ionized donors concentration in SCR (that lies along the interface plane) is directed oppositely to the gradient of dopant concentration in the GaAs bulk. So the nonequilibrium charge carriers produced by illumination in SCR of variable width $L_{\mathrm{SCR}}$ generate a photovoltage of opposite sign to that of the photovoltage generated by the charge carriers produced in the bulk.

There is a resistivity gradient in the GaAs bulk that is oriented parallel to the illuminated sample surface. Such a gradient may be modeled (as in the case of Ge-GaAs heterostructure) with slopes of both the conduction band bottom, $E_{C}$, and valence band top, $E_{V}$, levels.

In Fig. 4 a smooth $\mathrm{L}_{\mathrm{SCR}}$ change in the illuminated region (whose length is 1 ) is shown, by convention, with a dashed slanting line, according to the slopes of the valence band top and conduction band bottom levels in GaAs. By "plus" we labeled the charged donor atoms in the depletion region near the Schottky contact interface.

If the $\mathrm{NbN}-\mathrm{GaAs}$ sample is illuminated on the GaAs side with monochromatic light whose wavelength $\lambda=0.88 \mu \mathrm{m}$ (the corresponding absorption coefficient in $\mathrm{GaAs}$ is $\left.\alpha=800 \mathrm{~cm}^{-1}[18]\right)$, then the nonequilibrium charge carriers are produced in the GaAs bulk far from the interface (the GaAs substrate thickness $d$ is $200 \mu \mathrm{m}$ ) and move in the field of charged dopants concentration gradient parallel to the interface.

The experimentally found photovoltage polarity is determined by the above gradient (Fig. $3, \lambda=0.4-0.88 \mathrm{~mm}$ ). When the light wavelength is close to that corresponding to the GaAs intrinsic absorption edge, then charge carriers are produced both in the whole bulk and near the $\mathrm{NbN}$ GaAs interface (where the near-surface field oriented along the interface is opposite to the field in the bulk). The photovoltage generated in SCR becomes predominant. Due to this fact the sign of the photovoltage spectral curve is reversed at $\lambda>0.89 \mu \mathrm{m}(h v<1.39 \mathrm{eV})$ (see Fig. 3, curve 1).

At photon energies below the GaAs gap $E_{G}$ presence of photovoltage is related to the charge carriers production from the surface levels [19] (see Fig. 3, curves 1-3, $\lambda=0.89 \mu \mathrm{m}$ ).

An additional illumination with $\lambda=0.88 \mu$ m causes occurrence of photoconductivity and also the photovoltage amplitude drop in the $\lambda=\left(\begin{array}{lll}0.64 & 0.84\end{array}\right) \mathrm{mm}$ range (as in the GeGaAs heterostructure)

Presence of a longitudinal field in SCR may result in: (i) a decrease in SCR width; (ii) smoothing the band bending nonuniformity at the metal-semiconductor interface (in Fig. 4 SCR at illumination is shown with a dashed line that is parallel to the $\mathrm{NbN}-\mathrm{GaAs}$ interface); (iii) an increase in the recombination flow of the nonequilibrium charge carriers towards the interface. All the above factors will decrease a contribution from photocurrent (flowing along the interface) to the total photovoltage [20]. In this case the photovoltage polarity is determined predominantly by that of the bulk-gradient voltage over the whole range measured (see Fig. 3, curve 2). An additional illumination with 
photons whose energy $h v=1.35 \mathrm{eV}$ (i.e. $\lambda=0.92 \mu \mathrm{m}$ ) is below the GaAs gap does not result in appearance of an additional field in a sample. No photoconductivity appears in this case, so it cannot be the reason for a decrease in photovoltage amplitude.

A slight increase of the photovoltage amplitude near in the spectral region of $\lambda=0.85 \mu \mathrm{m}(h \nu=1.46 \mathrm{eV})$ may be related to generation of charge carriers from surface levels by the additional illumination. This leads to conductivity growth in SCR near the GaAs surface and, correspondingly, increases a contribution from the lateral photovoltage.

The photovoltage amplitude dependence on the intensity of exciting radiation has some peculiarities.

To illustrate, if (without additional illumination) the modulated radiation intensity is changed, then the voltage amplitude changes too. It should be noted that in this case both the form and sign of the corresponding portions of spectral characteristic do not change. The voltage amplitude does not change proportionally to the light intensity. When the intensity of modulated radiation is doubled, then the voltage amplitude is increased by a factor of about 1.5. This may serve as indication at presence of contribution from the «planar» voltage to the signal measured.

At the same time additional illumination of the sample with unmodulated radiation whose intensity is equal to that of modulated radiation (Fig. 3, curve 2), i.e., a twofold increase of intensity, changes the form of spectral characteristic rather than the voltage amplitude. From this one can conclude that effect of direct voltage generated by unmodulated additional illumination on the conditions of current flow in the structure studied is qualitatively different.

An additional evidence that the features of spectral characteristic of the voltage studied are related to band bending nonuniformity at the $\mathrm{NbN}-\mathrm{GaAs}$ contact lies in the fact that they depend on thermal annealing [21]. After the structure is annealed, its spectral characteristic is a curve with a pronounced peak near the GaAs absorption edge $(\lambda=0.89 \mu \mathrm{m})$ and abrupt drop of amplitude at both sides of the peak (i.e., this curve is similar to curve 2 in Fig. 3).

It is evident that both thermal annealing and unmodulated additional illumination result in a decrease of the band bending nonuniformity at the Schottky contact interface.

Another evidence of a drop of band bending nonuniformity is that unmodulated additional illumination does not affect the form of the spectral characteristic of voltage, chaging its amplitude but slightly.

\section{Conclusions}

The presented results prove that the technique at which a semiconductor structure is excited by concurrent action of modulated and unmodulated radiation is informative when studying layered structures, heterostructures and metal-semiconductor contacts.
The effect of transverse photovoltage generation in the structures studied serves as a typical demonstration of interaction between the photocurrents arising in their different layers.

The term «transverse photovoltage» is used here as a generalized one. From the above results it is obvious that a signal measured at the sample contacts is an algebraic sum of several voltages (that are generated in different layers of the structure studied and are characterized by different generation mechanisms). These are (i) bulk voltage related to nonuniform distribution of impurities; (ii) voltage due to nonuniformity of the transition layer; (iii) «planar» voltage related to band bending nonuniformity at the interface or semiconductor surface, as well as to asymmetric position of the illuminated sample area relative to the measuring contacts.

All the above voltages have, however, a common feature. They are generated (or have a component) along the direction that is normal to the gradient of concentration of the nonequilibrium charge carriers that are moving from the illuminated surface to the sample bulk. So one can conclude that use of the term «transverse voltage» when dealing with a signal measured in our experiments is quite correct and justified from the physical viewpoint.

For the Ge-GaAs heteroepitaxial structure an interaction between the currents resulting from photoconductivity and photovoltage leads to changes in both the spectral curves form and photovoltage sign. This is due to the fact that resistivity gradients near the interface are oriented oppositely in the Ge film and GaAs substrate.

A direct field generated in the film and substrate by an unmodulated additional illumination affects the spectral curve of the transverse photovoltage.

For Schottky contacts where depletion band bending is pronounced at metal-semiconductor interfaces the interaction between photocurrents flowing in the bulk and SCR is complicated too. A continuous field generated by an additional illumination and oriented along the metalsemiconductor interface leads to the decrease in SCR width, smoothing band bending and an increase in the recombination rate for electron-hole pairs at the metalsemiconductor interface. In this case a contribution to the total photovoltage (due to illumination with modulated light) from the photocurrent flowing in this region decreases.

For semiconductor heterostructures and Schottky contacts not only the spectral characteristics of transverse photovoltage are similar. Their features stem from the same physical reason, namely, nonuniform distribution of the dark charge carriers in the semiconductor bulk, near interface or near surface.

The principal conceptual conclusion from the work is as follows. In semiconductor structures with concentration nonuniformities in the bulk and at interfaces, unmodulated light generates nonequilibrium charge carriers and an additional direct field (voltage). This results in changing the form of spectral characteristic for the transverse photovoltage. 


\section{L.V. Shekhovtsov: Some features of transverse photovoltage...}

Another conclusion is that the transverse photovoltage measured in the above structures is a complicated algebraic sum resulting from photocurrents mechanisms of which are different.

Hence an analysis of the spectral characteristic form for the total photocurrent allows one to determine the area of a semiconductor structure where the light absorption occurs, as well as investigate the uniformity degree close to the interface.

This is the principal feature of the presented technique for investigation of composite semiconductor structures.

\section{Acknowledgment}

The author would like to thank R.V. Konakova and V.G. Lyapin for valuable discussions.

\section{References}

1. H.Palm, M.Arbes, and M.Schulz, Fluctuations of the AuSi(100) Schottky Barrier Height //Phys.Rev.Lett. 71, pp.22242227 (1993).

2. T.Sumiya, T.Miura, H.Fujinuma, and Sh.Tanaka, Schottky barrier inhomogeneity at $\mathrm{Au} / \mathrm{Si}(111)$ interfaces using ultrahighvacuum ballistic electron emission microscopy // Appl.Surf.Sci. 117-118, pp.329-333 (1997).

3. K.Maeda, and E.Kitahara, Metal-induced gap states model of nonideal Au/Si Schottky barrier with low defect density / IAppl.Surf.Sci. 130-132, pp.925- 929 (1998).

4. F.Gozzo, M.Marsi, H.Berger, G.Margaritondo, A.Ottolenghi, A.K.Ray-Chaudhuri, W.Ng, S.Liang, S.Singh, J.T.Welnak, J.P.Wallace, C.Capasso, and F.Cerrina, Microscopic-scale lateral ingomogeneities of the Schottky-barrier-formation process //Phys.Rev.(B) 48, pp.17163-17167 (1993).

5. M.Zacchigna, H.Berger, L.Sirigu, G.Margaritondo, G.F.Lorusso, H.Solak, and F.Cerrina, A spectromicroscopy study of the $\mathrm{Al} / \mathrm{GaS}$ interface: Evidence of band bending lateral inhomogeneities //J. of Electron Spectroscopy and Related Phenomena 101-103, pp.671-675 (1999).
6. J.Almeida, G.Margaritondo, C.Coluzza, S.Davy, M.Spajer, and D.Courjon, Laterally-resolved study of the $\mathrm{Au} / \mathrm{SiN}_{\mathrm{X}} /$ GaAs(100) interface //Appl.Surf.Sci. 125, pp.6-10 (1998).

7. Young Pil Kim, Si Kyung Choi, Hyun Kyong Kim, and Dae Won Moon, Direct observation of the Si lattice strain and its distribution in the $\mathrm{Si}(001)-\mathrm{SiO}_{2}$ interface transition layer // Appl.Phys.Lett. 71, pp.3504-3506 (1997).

8. Changyoung Kim, Paul L.King, and P.Pianetta, Fermi-level inhomogeneities on the GaAs(110) surface imaged with a photoelectron microscope //J.Vac.Sci.Technol.(b) 10, pp.1944-1948 (1992)

9. V.B.Bondarenko, Yu.A.Kudinov, S.E.Ershov, and V.V.Korablev, Natural the potential fluctuations at a doped semiconductor surface //FTP, 30, pp.2068-2075 (1996).

10. J.Tauc. Photo- and Thermoelectric Phenomena in Semiconductors (Russian translation). Mir, Moscow, 1962.

11. M.Alonso, R.Cimino, and K.Horn, Surface Photovoltage Effects in Photoemission from Metal-GaP(110) Interfaces: Importance for Band-Bending Evaluation //Phys.Rev.Lett. 64, pp.1947-1950 (1990).

12. D.Mao, A.Kahn, G.Le Lay, M.Marsi, Y.Hwu, and G.Margaritondo, Kelvin probe and synchrotron radiation study of surface photovoltage and band bending at metal/GaAs(100) interfaces // Appl.Surf.Sci. 56-58, pp.142-150 (1992).

13. L.V.Shekhovtsov, A.V.Sachenko, and Yu.I.Shwarts, Transverse photo-emf in a heteroepitaxial structure// FTP, 29, pp.566-573 (1995)].

14. D.E.Aspnes, and A.A.Studna, Dielectric functions and optical parameters of $\mathrm{Si}, \mathrm{Ge}, \mathrm{GaP}, \mathrm{GaAs}, \mathrm{GaSb}$, InP, InAs, and InSb from 1.5 to $6.0 \mathrm{eV}$ //Phys.Rev.(B) 27, pp.985-1009 (1983).

15. S.M.Ryvkin. Photoelectric Phenomena in Semiconductors (in Russian). GIFML, Moscow, 1963.

16. J.T.Wallmark, A New Semiconductor Photocell Using Lateral Photoeffect//Proc.IRE 45,pp.474-483(1957).

17. I.Hotovy, J.Brcka, and J.Huran, Investigation of Reactively Sputtered NbN Films //Fizika (A) 4, pp.337-342 (1995).

18. M.D.Sturge, Optical absorbtion of gallium arsenide between 0.6 and $2.75 \mathrm{eV} / /$ Phys.Rev. 127, pp.768-773 (1962).

19. F.Bechstedt, and R.Enderlein, Semiconductor Surfaces and Interfaces, Akademie-Verlag, Berlin, 1988.

20. V.N.Ovsyuk, Electronic Processes in Semiconductors with Space Charge Region (in Russian). Nauka, Novosibirsk, 1984.

21. L.V.Shekhovtsov, The Real Band Diagram for the Metal-Semiconductor Heterojunction //Proc.22-nd Int.Conf.on Microelectronics (MIEL-2000, Nis, Yugoslavia) 2, pp.193-195 (2000). 